\title{
CONTRIBUCIÓN DEL PERÚ EN LAS INICIATIVAS PARA PROMOVER LA SEGURIDAD SANITARIA MUNDIAL
}

\author{
Ernesto Gozzer ${ }^{1, a}$, Fredy Canchihuamán ${ }^{1, b}$, Marina Piazza ${ }^{1, \mathrm{c}}$, Hans Vásquez $^{1, \mathrm{~d}}$, Gisely Hijar ${ }^{1, \mathrm{e}}$, \\ Aníbal Velásquez,a
}

\begin{abstract}
RESUMEN
El artículo describe el concepto de la seguridad sanitaria mundial, su evolución en el tiempo y discute la importancia de enfrentar los problemas que amenazan la salud global producidas por agentes biológicos de manera natural, accidental o intencional. Asimismo, presenta las iniciativas surgidas en las últimas dos décadas en el mundo, y el rol del Perú en el desarrollo de la Agenda para La Seguridad Sanitaria Mundial o Global Health Security Agenda (GHSA) y la Alianza para las Evaluaciones Externas. Al respecto, el Perú ha cumplido un papel importante participando con un grupo de países, la Organización Mundial de la Salud (OMS) y la GHSA en el proceso de desarrollo de un instrumento de evaluación externa conjunta denominado Joint External Evaluation Tool (JEE) con el objetivo último de acelerar la implementación del Reglamento Sanitario Internacional (RSI).
\end{abstract}

Palabras clave: Medidas de Seguridad; Salud global; Evaluación; Cooperación Internacional; Perú; Brotes/prevención y control (fuente: DeCS BIREME).

\section{CONTRIBUTION OF PERU IN INITIATIVES TO PROMOTE GLOBAL HEALTH SECURITY}

\begin{abstract}
This article describes the concept of global health security and its development over time. It emphasizes the need to control challenges against global health such as those produced by biologic agents both natural and intentional. It presents world initiatives that have developed during the last two decades and the contribution of Peru to the Global Health Security Agenda (GHSA) and the Monitoring and Evaluation Framework. In order to accelerate the implementation of the International Health Regulations Peru has played an important role to accelerate the implementation of International Health Regulations (IHR), participating with a group of countries, World Health Organization (WHO), and GHSA in the creation of the Joint External Evaluation Tool (JEE).
\end{abstract}

Key words: Security Measures; Global health; Evaluation; International Cooperation; Peru; Disease outbreaks/prevention \& control (source: MeSH NLM).

\section{EMERGENCIA DE LA SALUD EN EL CAMPO DE LA SEGURIDAD SANITARIA MUNDIAL}

El concepto de seguridad en salud tiene sus orígenes el siglo XIV, cuando se establecen cuarentenas como medidas para evitar la propagación de la peste bubónica en países europeos. A mediados del siglo XIX la salud pública ingresa como tema de discusión sobre seguridad nacional en el campo de las relaciones diplomáticas, sumándose a los temas de comercio internacional y de acciones militares que eran los que prevalecían. A finales del siglo XX se empieza a considerar la propagación de enfermedades infecciosas como amenazas a la seguridad nacional de orden no militar ${ }^{(1,2)}$. En 1969, el Reglamento
Sanitario Internacional (RSI) o International Health Regulations (IHR) es adoptado por la Asamblea de la Salud como el instrumento que, desde un marco legal, establece lineamientos vinculados con la seguridad internacional con el objetivo de prevenir la propagación de enfermedades y afrontar amenazas a la salud pública ${ }^{(3)}$.

Durante las últimas tres décadas las relaciones entre los países se han transformado aceleradamente dando lugar a la globalización. Para Held y McGrew ésta Designa la escala ampliada, la magnitud creciente, la aceleración y la profundización del impacto de los flujos y patrones transcontinentales de interacción social (4). Estos cambios tienen un impacto positivo y negativo sobre la salud. La rápida interconexión entre personas y bienes de la economía global permite también la rápida

\footnotetext{
Instituto Nacional de Salud. Lima, Perú.

Ministerio de Salud. Lima, Perú.

Médico cirujano MSc, ${ }^{\mathrm{b}}$ médico cirujano MPH PhD, ${ }^{\mathrm{c}}$ psicóloga clínica MPH, ScD, ${ }^{\mathrm{d}}$ médico cirujano MPH, ${ }^{\mathrm{e}}$ bióloga MSc Recibido: 19/05/2016 Aprobado: 15/06/2016
} 
propagación de las enfermedades y otros daños para la salud, generando una situación de vulnerabilidad.

La seguridad en salud es propuesto como un nuevo paradigma que puede permitir vidas más longevas y plenas, y contribuir, a su vez, a la sostenibilidad de las mejoras socioeconómicas. La seguridad en salud, que es simplemente la protección de las amenazas a la salud, es un concepto amplio que incluye la seguridad colectiva e individual, la primera orientada a la prevención, detección y respuesta rápida en el ámbito mundial y la segunda a brindar acceso a servicios de salud, productos y tecnologías seguros y efectivos

A pesar de los grandes avances en el conocimiento y la tecnología en salud para afrontar los riegos o amenazas en salud pública, todavía existen grandes brechas en términos de recursos humanos, capacidades para la vigilancia y, especialmente, para la coordinación de las intervenciones. De hecho, no existe un sistema global articulado para prevenir epidemias, detectar brotes en tiempo real, compartir información y muestras de laboratorio que permitan responder oportunamente a esas amenazas. Esta debilidad puede impactar tremendamente en la salud, la economía y la estabilidad de un país, como ocurrió en la limitada respuesta global a la epidemia de ébola en Guinea, Sierra Leona y Liberia ${ }^{(6,7)}$.

\section{¿POR QUÉ ES NECESARIA LA SEGURIDAD SANITARIA MUNDIAL?}

\begin{abstract}
En las últimas dos décadas han emergido y reemergido varias enfermedades infecciosas con potencial pandémico que amenazan la salud pública como la influenza aviar H5N1 en 1997 y 2003; el virus del Nilo Occidental en 1999; el síndrome respiratorio agudo y grave (SARS), en el 2002; la epidemia de chikungunya en Kenya, en 2004, India 2005 y las Américas 2013; la influenza H1N1 en 2009, y el síndrome respiratorio de oriente medio (MERS CoV) en $2012^{(8-11)}$. De igual manera, ha surgido la actual epidemia del Zika en las Américas iniciada en el 2015.
\end{abstract}

La epidemia del Zika, extendida en poco más de un año a más de 38 territorios o países de las Américas, revela la importancia de contar con mecanismos globales ágiles y efectivos para que los países se preparen y respondan a estas graves amenazas a la salud pública ${ }^{(12-14)}$. Además de los costos humanos y económicos del manejo de las epidemias y daños a la salud pública, el Perú en particular tiene algunas características que lo podrían hacer más vulnerable a estos eventos como un complejo perfil epidemiológico en el que persisten las enfermedades transmisibles y al que se agregan las enfermedades no transmisibles, una biodiversidad que podría ser afectado por las amenazas que plantea el cambio climático y una ubicación geográfica en zona de potenciales desastres.
El costo en términos de vidas humanas que se pierden o afectan por las epidemias, el gasto en sus medidas de control y su impacto sobre la economía global es muy alto. Las epidemias pueden modificar, además, los comportamientos e impactar indirectamente en otras áreas de la economía como el turismo, comercio, entre otros. Lee y McKibbin estimaron que el costo de la epidemia de SARS del 2003, pudo alcanzar los 54 mil millones de dólares ${ }^{(15)}$. La epidemia del cólera de 1991 que empezó en nuestro país y luego se expandió a 35 países de Las Américas y África causó 2909 muertes y pérdidas por 495,3 millones de dólares ${ }^{(16,17)}$. La reciente epidemia del ébola se estima que ocasionó gatos directos y pérdidas económicas por aproximadamente 6 y 15 billones de dólares, respectivamente ${ }^{(18)}$.

La seguridad de la salud global también considera las amenazas intencionales a la salud humana, animal, vegetal y a la economía de los países y su abordaje requiere un enfoque integrador que articule los esfuerzos de diferentes disciplinas y organizaciones ${ }^{(19)}$. Considerar a la seguridad de la salud global en un sentido más amplio, permitirá fortalecer tanto la capacidad global como la de los países para prevenir, detectar y responder también frente a las amenazas antes descritas.

\section{ESTRATEGIAS PARA EL DESARROLLO DE LA SEGURIDAD SANITARIA MUNDIAL}

En la Convención Sanitaria Internacional del año 1926, se acordaron medidas para uniformizar intervenciones orientadas a prevenir la propagación de las epidemias relacionadas al comercio marítimo. Desde entonces, se ha realizado una serie de convenciones y elaborado diferentes versiones del denominado Reglamento Sanitario Internacional (RSI) siendo su más reciente versión la del 2005 que entró en vigencia en el año $2007^{(3,20) "}$. En su artículo segundo señala que la finalidad es: Prevenir la propagación internacional de enfermedades, proteger contra esa propagación, controlarla y darle una respuesta de salud pública proporcionada y restringida a los riesgos para la salud pública y evitando al mismo tiempo las interferencias innecesarias con el tráfico y el comercio internacionales. EI RSI establece asimismo la obligación de los Estados de realizar las acciones necesarias para fortalecer sus capacidades de detectar, evaluar y notificar eventos de acuerdo a lo establecido en el reglamento en un plazo no mayor de cinco años desde su entrada en vigencia. No obstante estos esfuerzos, en el 2012 se determinó que solo cerca del $20 \%$ de los países cumplió con los requisitos establecidos en el RSI, para prevenir, detectar y responder rápidamente frente a las amenazas que representan las epidemias (21). Revisiones respecto al RSI han resultado en recomendaciones sobre estrategias para acelerar su implementación y mejorar su funcionamiento que incluyen entre otros las evaluaciones externas ${ }^{(22)}$. 
En el 2014, se crea La Agenda para la Seguridad Sanitaria Mundial o Global Health Security Agenda (GHSA) para responder a las amenazas naturales, accidentales o intencionales - a la salud pública y a la seguridad, global, regional y nacional ${ }^{23)}$. La GHSA es: Una iniciativa de países, organismos internacionales y sociedad civil que busca acelerar el avance hacia un mundo más seguro y protegido de las amenazas de las enfermedades infecciosas; promover la seguridad de la salud global como una prioridad internacional; y promover la completa implementación del Reglamento Sanitario Internacional 2005 (RSI), la Evaluación del Desempeño de los Servicios Veterinarios (PVS) de la Organización Mundial de Sanidad Animal (OIE) y otros enfoques relevantes para la seguridad de la salud global (24).

Con el fin de orientar la evaluación de las capacidades de los países en seguridad global en salud y la implementación de acciones y compromisos para reducir las brechas producto de la evaluación, la GHSA identificó once áreas en seguridad global o paquetes de acción. Asimismo, promovió la realización de evaluaciones externas entre los países miembros, con el fin de establecer una línea de base, identificar las brechas y medir el avance en estas 11 áreas o paquetes de acción. Para ello, elaboró una herramienta de evaluación que fue validada en cinco países durante el año 2015: Georgia, Perú, Portugal, Uganda y Reino Unido que fue antecedida en cada país por una evaluación interna.

Perú integra, con otros 49 países, la GHSA, que es conducida por un Comité Directivo formado por 10 países. Como miembros asociados participan la Organización Mundial de la Salud (OMS), la Organización de las Naciones Unidas para la Agricultura y Alimentación (FAO), la Organización Mundial de Salud Animal (OIE), la Comunidad Económica de Estados de África Occidental (ECOWAS), Interpol, la Organización de Naciones Unidas para la Reducción de Desastres (UNISDR) y la Unión Europea.

Al fundarse en 2014, se acordó apoyar a África para enfrentar la epidemia de ébola, y apoyar a los países con mayores brechas. EE.UU. anunció que apoyaría al menos a 30 países miembros y que invertiría mil millones de dólares en cinco años para apoyar los planes de acción. En la segunda reunión en el 2015 se hizo un llamado a los países participantes para profundizar acciones para fortalecer sus sistemas de salud y acelerar el total cumplimiento del RSI, el PVS y otros acuerdos relacionados con la seguridad sanitaria mundial. Asimismo, se estableció una plataforma de colaboración entre los países que participan en las evaluaciones externas en tanto se ajustan las herramientas de monitoreo y evaluación de la implementación del RSI. La GHSA promueve la cooperación regional e internacional en concordancia con las Metas de Desarrollo Sostenible.

\section{ALIANZA PARA LAS EVALUACIONES EXTERNAS Y USO DE LA HERRAMIENTA DE EVALUACIÓN EXTERNA CONJUNTA}

En octubre del 2015, un grupo técnico convocado por la OMS sobre el marco del monitoreo y evaluación del Reglamento Sanitario Internacional (RSI), recomendó el desarrollo de un proceso de evaluaciones y una herramienta para evaluaciones externas conjuntas, considerando que las evaluaciones externas por pares pueden ser un catalizador de la implementación del RSI. En base a las recomendaciones, la OMS, con los resultados de las experiencias en los países piloto que aplicaron la herramienta GHSA con once paquetes, publicó una nueva herramienta más amplia de evaluación denominada evaluación externa conjunta o Join External Evaluation (JEE). Esta herramienta tiene 19 Áreas Técnicas que incluyen la totalidad de los once paquetes de acción de GHSA y todos los elementos relevantes para el Reglamento Sanitario Internacional (RSI), incluyendo las áreas consideradas en el instrumento de evaluación de capacidades básicas del RSI, de tal modo que exista una única herramienta de evaluación externa ${ }^{(24,25)}$. La herramienta JEE considera como elementos clave, la prevención, detección y respuesta, y ayuda a determinar las capacidades básicas, las brechas y necesidades, el desarrollo de los planes u hojas de ruta, monitorizar el progreso de la implementación de las acciones para enfrentar las amenazas a la salud pública en el marco del RSI y a generar información para el país, los colaboradores y financiadores ${ }^{(25)}$ (Tabla 1).

Para organizar y acelerar la realización de evaluaciones externas en los países, en marzo de 2016 se acordó crear una Alianza para las Evaluaciones Externas, cuya meta es realizar evaluaciones utilizando la herramienta JEE en 135 países en dos años. La Alianza estará dirigida por un Grupo Asesor y ejecutará sus acciones apoyada por el Secretariado asumido por la OMS. la Alianza facilitará el compromiso entre los países, las organizaciones internacionales, los donantes y los expertos técnicos que participan en el proceso de evaluación y promoverá la transparencia en el intercambio de los resultados de las evaluaciones, en particular, a los donantes interesados en financiar el desarrollo y la fortalecimiento de las capacidades de los países. El trabajo de la Alianza estará vinculado estrechamente con otros componentes de la vigilancia del RSI y la evaluación del enfoque (Figura 1).

\section{PARTICIPACIÓN DEL PERÚ EN LA INICIATIVA PARA MEJORAR LA SEGURIDAD SANITARIA MUNDIAL}

Desde el 2013, el Perú se encuentra implementando una reforma de salud la cual, entre otras prioridades, enfatiza la profundización de las acciones de promoción de la 
Tabla 1. Comparación de los instrumentos para evaluar las capacidades de los países en seguridad global en salud

\begin{tabular}{|c|c|}
\hline Instrumento GHSA & Instrumento JEE \\
\hline Paquetes de acción & Áreas técnicas \\
\hline \multicolumn{2}{|l|}{ Prevenir } \\
\hline Resistencia a los antimicrobianos & Legislación nacional, política y financiamiento \\
\hline Enfermedad zoonótica & Coordinación, comunicación y promoción del RSI \\
\hline Biocustodia/Bioseguridad & Resistencia a los antimicrobianos \\
\hline \multirow[t]{4}{*}{ Inmunización } & Enfermedad zoonótica \\
\hline & Inocuidad alimentaria \\
\hline & Biocustodia/Bioseguridad \\
\hline & Inmunización \\
\hline \multicolumn{2}{|l|}{ Detectar } \\
\hline Sistema nacional de laboratorios & Sistema nacional de laboratorios \\
\hline Vigilancia en tiempo real & Vigilancia en tiempo real \\
\hline Reporte GHSA & Reporte \\
\hline Desarrollo de la fuerza laboral & Desarrollo de la fuerza laboral \\
\hline \multicolumn{2}{|l|}{ Responder } \\
\hline & Preparación \\
\hline Centros de operaciones de emergencia & Operaciones de respuesta a emergencias \\
\hline Vinculación de salud pública y aplicación de la ley & Vinculación de salud pública y autoridades de seguridad \\
\hline \multirow[t]{2}{*}{ Medidas de protección médicas y despliegue del personal } & Medidas de protección médicas y despliegue del personal \\
\hline & Comunicación de riesgo \\
\hline \multicolumn{2}{|l|}{ Otros riesgos relacionados al RSI y puntos de entrada } \\
\hline & Puntos de entrada \\
\hline & Eventos químicos \\
\hline & Emergencias causadas por radiación \\
\hline
\end{tabular}

GHSA: Global Health Security Agenda; JEE: Joint External Evaluation Tool; RSI: Reglamento Sanitario Internacional

salud fomentando estilos de vida y entornos saludables, así como las de prevención y control de enfermedades, que van desde las inmunizaciones hasta el control ambiental. La reforma busca asegurar recursos para que los servicios de salud estén preparados y puedan atender las situaciones de epidemias y desastres, y mitigar sus potenciales daños y controlar los efectos ${ }^{(26)}$.

En este contexto, el Perú, que realizaba algunos avances en la implementación del RSI, decide participar en la GHSA con el objetivo de mejorar su capacidad de respuesta para enfrentar las amenazas a la seguridad sanitaria mundial. En septiembre de 2014, en la reunión de alto nivel de la GHSA, el ministro de Salud propuso a Perú como uno de los cinco países voluntarios para realizar la validación de la herramienta de autoevaluación elaborada por la GHSA, así como asumir el liderazgo y abogacía en dos paquetes de acción, sobre respuesta multisectorial y sobre bioseguridad y biocustodia, y colaborar en ocho de ellos. Como consecuencia de esta participación, en el Perú se han elaborado materiales de discusión y en el Instituto Nacional de Salud se ha reforzado el área de bioseguridad e iniciado la elaboración de planes de desarrollo en bioseguridad y biocustodia.
En enero de 2015, una misión de evaluación externa enviada por GHSA visitó el Perú. La preparación, realización y evaluación de la visita fue liderada por el Dr. Ernesto Gozzer (MINSA) y el Dr. Ernesto Bustamante (INS). Este proceso incluyó la realización de teleconferencias con expertos de GHSA y talleres de autoevaluación con la participación de funcionarios y expertos de sectores como: Ambiente, Agricultura, Defensa, Energía y Minas, Ministerio Público, Presidencia del Consejo de Ministros, Producción, Interior, Relaciones Exteriores, Salud y Transportes y Comunicaciones. La misión realizó entrevistas, mesas redondas y otras reuniones, y visitó el Centro de Operaciones de Emergencia del MINSA y el laboratorio de Biomedicina BSL2/BSL3 del INS en Chorrillos.

Los puntajes de la autoevaluación coincidieron con los de la misión en 22 de los 24 indicadores. Se encontró que en algunos paquetes de acción como el de inmunizaciones y vigilancia epidemiológica, los puntajes eran altos. Por el contrario en los correspondientes a biocustodia y la relación entre salud animal y salud pública los puntajes resultaron bajos. El equipo peruano realizó sugerencias para precisar mejor las escalas de valoración de varios indicadores. 


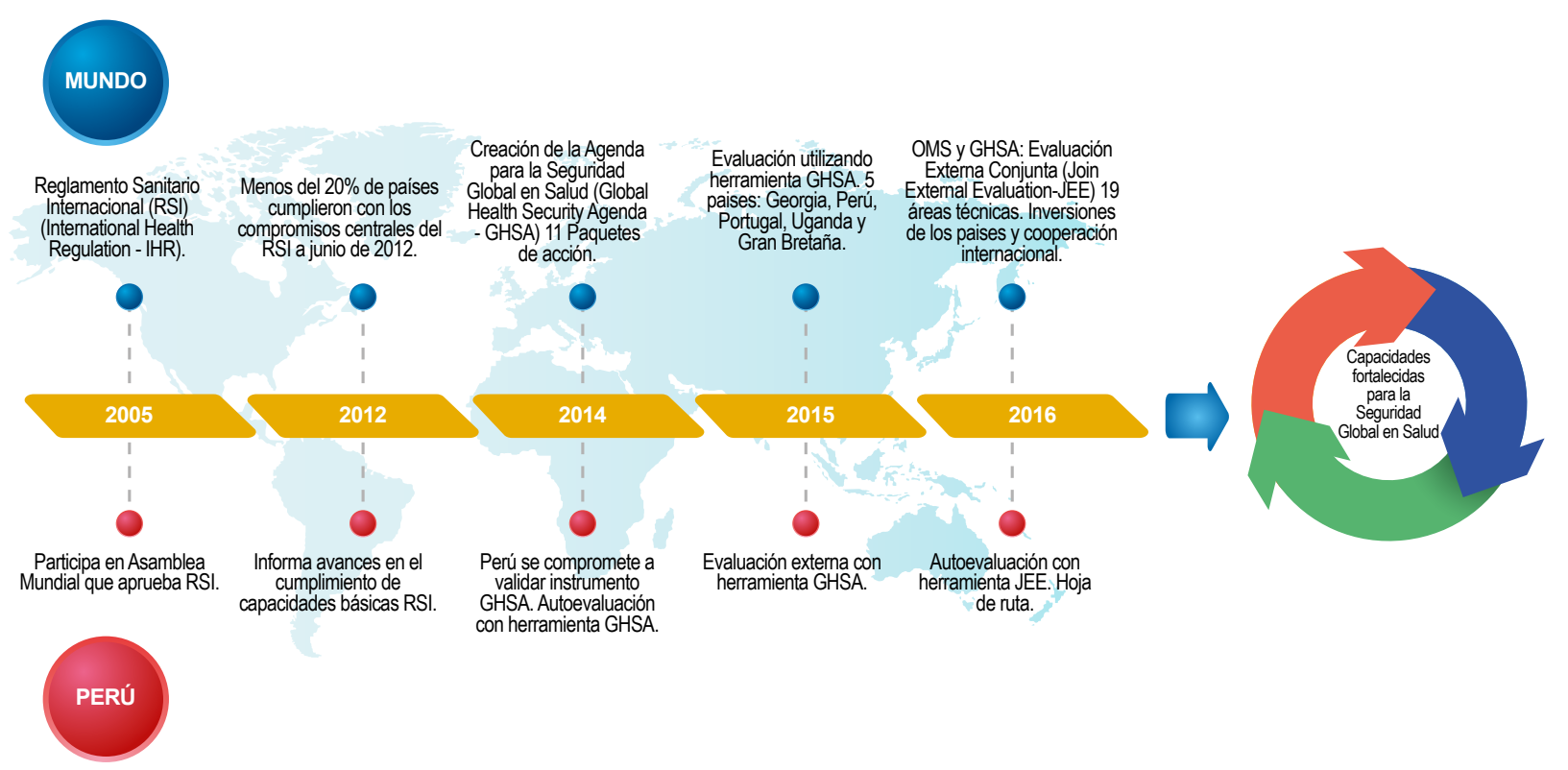

Figura 1. Desarrollo de las iniciativas para fortalecer la seguridad global en el mundo y en el Perú

También se propuso separar algunos indicadores como el de capacidad de respuesta a las amenazas en el que se mezclaba la respuesta a epidemias, en los que Perú tiene experiencia, con la respuesta a bioterrorismo, en los que no existe mucho desarrollo. El informe detallado fue enviado a GHSA ${ }^{(24)}$ y varios de esos aportes han sido usados para la elaboración de la herramienta JEE.

El Perú ha presentado la experiencia y aportes en varios foros de GHSA. En la Reunión de Alto Nivel de Seúl, Corea del Sur en septiembre de 2015 se confirmó la necesidad de promover la realización de evaluaciones externas en otros países y, así como la reunión de alto nivel realizada en Ginebra en marzo de 2016. En esta ocasión se decidió la creación de la Alianza para Evaluaciones Externas, se confirmó la utilización de JEE como nuevo instrumento conjunto y se estableció la meta de realizar evaluaciones externas voluntarias en 135 países.

El Perú ha completado una autoevaluación de los ocho paquetes de acción -ahora áreas técnicas- que no fueron parte de la evaluación piloto realizada por la GHSA. A partir de esta, se ha elaborado una hoja de ruta que ha sido revisada por una misión de evaluadores externos para luego preparar un plan de acción. En esta ocasión además de los sectores ya mencionados han participado representantes de organismos de cooperación como OPS/OMS y USAID. En todas las etapas los representantes de los sectores involucrados, han asumido la responsabilidad de liderar las áreas técnicas de acuerdo a su ámbito de competencia. La posición de Perú y su capacidad de colaborar en el campo de la seguridad sanitaria mundial ha llevado a que el Perú haya participado en la evaluación externa de Estados Unidos, liderando uno de las áreas técnicas a evaluar.

La participación del Perú en la GHSA permitió analizar con mayor profundidad las brechas en su capacidad de preparación y respuesta y acelerar los procesos institucionales y técnicos. Esto ha contribuido a mejorar la respuesta a epidemias que amenazaban ser incontrolables como el chikungunya, y que en el Perú causó menos de 300 casos a diferencia de los cientos de miles que se produjeron en otros países de la región, y que se retrasará el ingreso del zika, que se ha presentado en un tiempo mayor a un año después de haberse reportado en Brasil. Para consolidar lo alcanzado, el MINSA formuló en 2016, la Ley 30423, que fortalece su autoridad para proteger la seguridad de la salud nacional dado que hace posible intervenir ante situaciones de riesgo para la vida y la salud antes que se produzcan epidemias y emergencias por incumplimiento de funciones de los gobiernos o factores exógenos.

\section{CONCLUSIONES}

En las últimas décadas se ha producido una aceleración de la globalización, y también han emergido y reemergido amenazas a la seguridad de la salud en el mundo que requiere la participación de países, organismos multilaterales y sociedad civil con un enfoque multisectorial y multidisciplinario para vivir en un mundo más seguro.

La Agenda para la Seguridad Sanitaria Mundial (GHSA) es una iniciativa de países y organismos que busca acelerar los procesos de mejora de capacidades de los países para responder a las amenazas a la salud global que promueve el uso de una plataforma colaborativa y las evaluaciones externas voluntarias. La GHSA y la OMS se han unido para desarrollar una nueva herramienta de evaluación externa conjunta (JEE), la cual será utilizada en la evaluación de capacidades de 135 países en dos años, con el fin de implementar el Reglamento Sanitario Internacional. Para ello se ha creado, en marzo de 2016, una alianza para las evaluaciones externas. 
La respuesta global es fundamental para hacer frente a estas amenazas a la seguridad de la salud pública global; la participación del Perú en el GHSA ha fortalecido sus capacidades nacionales y de cooperación con otros países. Una muestra de ello es la respuesta que se ha dado frente a los brotes de chikungunya y zika. La participación multisectorial de diferentes organismos del Estado ha sido fundamental para el desarrollo de estas iniciativas. EI Perú actualmente está colaborando con la evaluación de capacidades de otros países. En el ámbito internacional se ha reconocido esta contribución y la experiencia peruana es citada en los foros en los que se construye este esfuerzo global.

Contribuciones de los autores: EG, FC, MP, HV y GH participaron
en la concepción del trabajo. EG, escribió la primera versión del
manuscrito. Todos los autores aportaron a la redacción y revisión crítica del manuscrito y su aprobación final.

Fuentes de financiamiento: autofinanciado

Conflictos de interés: los autores declaran no tener conflictos de interés en la publicación del artículo.

\section{REFERENCIAS BIBLIOGRÁFICAS}

1. Fidler D. Public Health and National Security in the Global Age: Infectious Diseases, Bioterrorism, and Realpolitik [Internet]. Indiana: Indiana University Bloomington; 2003 [citado el 17 de mayo 2016]. Disponible en: http://www. repository.law.indiana.edu/facpub/416

2. BaylisJ,Smith S, editors. The Globalization of World Politics. An Introduction to International Relations. $6^{\text {th }}$ ed. Londres: Oxford University Press; 2014.

3. World Health Organization. International Health Regulations (2005). $2^{\text {nd }}$ ed. Ginebra: WHO; 2008.

4. Held D, McGrew A. Globalización, antiglobalización: sobre la reconstrucción del orden mundial. Barcelona: Paidós; 2003.

5. Heymann DL, Chen L, Takemi K, Fidler DP, Tappero JW, Thomas MJ, et al. Global health security: the wider lessons from the west African Ebola virus disease epidemic. Lancet. 2015;385(9980):1884-901. doi: 10.1016/S0140-6736(15)60858-3.

6. Lev O, Rager-Zisman B. Protecting public health in the age of emerging infections. Isr Med Assoc J. 2014;16(11):677-82.

7. Kekulé AS. Learning from Ebola Virus: How to Prevent Future Epidemics. Viruses. 2015;7(7):3789-97. doi: $10.3390 / \mathrm{v} 7072797$.

8. Morens DM, Fauci AS. Emerging Infectious Diseases: Threats to Human Health and Global Stability. PLOS Pathog. 2013;9(7):e1003467. doi: 10.1371/journal.ppat.1003467.

9. Saiz JC, Vázquez-Calvo Á, Blázquez AB, Merino-Ramos T, Escribano-Romero E, Martín-Acebes MA.Zika Virus: the Latest Newcomer. Front Microbiol. 2016;7:496. doi: 10.3389/fmicb.2016.00496.

10. Milne-Price S, Miazgowicz KL, Munster VJ. The emergence of the Middle East respiratory syndrome coronavirus. Pathog Dis. 2014;71(2):121-36. doi: 10.1111/2049-632X.12166.
11. Petersen LR, Powers AM. Chikungunya: epidemiology. F1000Research. 2016;5. doi: 10.12688/f1000research.7171.1.

12. Ippolito G, Di Caro A, Capobianchi MR. The Chronology of the International Response to Ebola in Western Africa: Lights and Shadows in a Frame of Conflicting Position and Figures. Infect Dis Rep. 2015;7(2):5957. doi: 10.4081/ idr.2015.5957.

13. Fellner C.Zika Virus: Anatomy of a Global Health Crisis. P T. 2016;41(4):242-53.

14. Pan American Health Organization, World Health Organization. Regional Zika Epidemiological Update (Americas) - 1 May 2016 [Internet]. Washington, D.C.: PAHO/WHO; 2016 [citado el 15 de mayo del 2016]. Disponible en: http://www.paho. org/hq/index.php?option $=$ com_co ntent \&view =article\&id=1 1599: re gional-zika-epidemiological-updateamericas\&Itemid $=416918$ lang $=$ en

15. Institute of Medicine (US) Forum on Microbial Threats; Knobler S, Mahmoud A, Lemon S, et al., editors. Learning from SARS: Preparing for the Next Disease Outbreak: Workshop Summary. Washington, D.C.: National Academies Press (US); 2004. Disponible en: http://www.ncbi.nlm.nih.gov/books/ NBK92475/

16. Petrera M. Impacto Económico de la Epidemia del Cólera Perú-1991. Lima: OPS/OMS; 1992.

17. World Health Organization. Global Health Atlas [Internet]. Geneva; WHO; 2003-2007 [citado el 1 de Diciembre del 2014]. Disponible en: http://apps.who. int/globalatlas/

18. Ross AGP, Crowe SM, Tyndall MW. Planning for the Next Global Pandemic. Int J Infect Dis. 2015;38:89-94. doi: 10.1016/j.jiji.2015.07.016.

19. Gronvall G, Boddie C, Knutsson R, Colby M. One health security: an important component of the global health security agenda. BiosecuR Bioterror. 2014;12(5):221-4. doi: 10.1089/ bsp.2014.0044.

20. White CF. Plague: Modern Preventive Measures in Ships and Ports. Proc R Soc Med. 1935;28(5):591-602.

21. Centersfor Disease Control and Prevention. Global Health Security [Internet]. Atlanta: CDC; 2016 [citado el 1 de diciembre del 2016]. Disponible en: http://www.cdc. gov/globalhealth/healthprotection/ghs/ index.html

22. Gostin LO, DeBartolo MC, Friedman EA. The International Health Regulations 10 years on: the governing framework for global health security. Lancet. 2015;386(10009):2222-6. doi: 10.1016/ S0140-6736(15)00948-4.

23. Katz R, Sorrell EM, Kornblet SA, Fischer JE. Global health security agenda and the international health regulations: moving forward. Biosecur Bioterror. 2014;12(5):231-8. doi: 10.1089/ bsp.2014.0038.

24. Global Health Security Agenda. AboutGlobal Health Security Agenda [Internet]. Helsinki: GHSA; 2014 [citado el 17 de mayo del 2016]. Disponible en: https:// ghsagenda.org/about.html

25. Global Capacities Alert and Response (GCR). Joint External Evaluation tool: International Health Regulations. Ginebra: WHO; 2016. p. 98.

26. Perú, Ministerio de Salud, Consejo Nacional de Salud. Lineamientos y medidas de reforma del sector salud [Internet]. Lima: MINSA; 2013 [citado el 17 de mayo de 2016]. Disponible en: http://www.minsa.gob.pe/portada/ Especiales/2013/reforma/documentos/ documentoreforma10102013.pdf

Correspondencia: Ernesto Gozzer

Dirección: Calle Cápac Yupanqui 1400. Jesús

María. Lima 11, Perú

Teléfono: (511) 748-1111 anexo

Correo electrónico:ernesto.gozzer@gmail.com 